\title{
A HOTAIR regulatory element modulates glioma cell sensitivity to temozolomide through long-range regulation of multiple target genes
}

\author{
Lei Zhang, ${ }^{1,5}$ Anshun He, ${ }^{1,5}$ Bohan Chen, ${ }^{1}$ Jinfang Bi, ${ }^{1}$ Jun Chen, ${ }^{1}$ Dianhao Guo, ${ }^{1}$ \\ Yuyang Qian, ${ }^{1}$ Wenbin Wang, ${ }^{1}$ Tengfei Shi, ${ }^{1}$ Zhongfang Zhao, ${ }^{1}$ Jiandang Shi, ${ }^{1}$ \\ Woojin An, ${ }^{2,3}$ Frank Attenello, ${ }^{3,4}$ and Wange $\mathrm{Lu}^{1}$ \\ ${ }^{1}$ State Key Laboratory of Medicinal Chemical Biology and College of Life Sciences, Nankai University, 300071, Tianjin, China; \\ ${ }^{2}$ Department of Biochemistry and Molecular Medicine, Norris Cancer Center, University of Southern California, Los Angeles, \\ California 90033, USA; ${ }^{3}$ Keck School of Medicine, University of Southern California, Los Angeles, California 90033, USA; \\ ${ }^{4}$ Department of Neurological Surgery, University of Southern California, Los Angeles, California 90033, USA
}

\begin{abstract}
Temozolomide (TMZ) is a frequently used chemotherapy for glioma; however, chemoresistance is a major problem limiting its effectiveness. Thus, knowledge of mechanisms underlying this outcome could improve patient prognosis. Here, we report that deletion of a regulatory element in the HOTAIR locus increases glioma cell sensitivity to TMZ and alters transcription of multiple genes. Analysis of a combination of RNA-seq, Capture Hi-C, and patient survival data suggests that CALCOCO1 and ZC3H1O are target genes repressed by the HOTAIR regulatory element and that both function in regulating glioma cell sensitivity to TMZ. Rescue experiments and $3 \mathrm{C}$ data confirmed this hypothesis. We propose a new regulatory mechanism governing glioma cell TMZ sensitivity.
\end{abstract}

[Supplemental material is available for this article.]

Glioma is the most common primary brain tumor, and $~ 80 \%$ of malignant primary tumors in the central nervous system are glioma (Weller et al. 2015). Glioblastoma (GBM) is the most aggressive glioma and constitutes $>50 \%$ of all gliomas. Despite treatment advances, prognosis for glioblastoma is still very poor, and median survival time for patients is only 14-17 mo (Reifenberger et al. 2017). Current standard treatment options include surgery, radiation, and chemotherapy, specifically temozolomide (TMZ), which is the most frequently used clinical chemotherapy for GBM (Wick et al. 2012; Sandmann et al. 2015). TMZ efficiently crosses the blood brain barrier and induces glioma cell apoptosis; however, chemoresistance often develops, representing a major therapeutic obstacle. Thus, strategies to increase glioma cell sensitivity to TMZ could serve as useful interventions to improve patient prognosis.

Glioma resistance to TMZ is associated with multiple factors. Some report that high expression of O-6-methylguanine-DNA methyltransferase (MGMT) is related to robust TMZ resistance (Kitange et al. 2009; Wick et al. 2014). In addition, factors such as P4HB (Sun et al. 2013), ALDH1A1 (Schäfer et al. 2012), and poly (ADP-ribose) polymerase (Clarke et al. 2009) have been associated with TMZ resistance in glioma cells. However, clinical tests of strategies designed to attenuate TMZ resistance have not yet produced satisfactory results (Quinn et al. 2009; Warren et al. 2012), requiring further investigations.

Zhang et al. recently identified a regulatory element in the locus of the long noncoding RNA HOTAIR (Zhang et al. 2014).

\footnotetext{
${ }^{5}$ These authors contributed equally to this work. Corresponding authors: joyleizhang@nankai.edu.cn, wangelv@gmail.com

Article published online before print. Article, supplemental material, and publication date are at http://www.genome.org/cgi/doi/10.1101/gr.251058.119.
}

That study suggested that this element functions as an enhancer associated with progression of esophageal squamous cell carcinoma (ESCC) (Zhang et al. 2014). Follow-up studies report that this regulatory element or single-nucleotide polymorphisms (SNPs) within it are associated with risk or progression of uterine cervical (Weng et al. 2018), head and neck (Pan et al. 2017), and lung (Wang et al. 2018) cancers, while few studies report association of this element with glioma.

Based on ChIP data reported by Rheinbay and colleagues (Rheinbay et al. 2013), we found that in glioma cells and in glioblastoma stem cells derived from human tumors, the HOTAIR regulatory element is marked by $\mathrm{H} 3 \mathrm{~K} 4 \mathrm{me} 1, \mathrm{H} 3 \mathrm{~K} 4 \mathrm{me} 3$, and H3K27me3. Overall, H3K4me1 marks candidate enhancers, and the H3K4me3 mark is an active mark of transcriptional initiation (Zhou et al. 2011; Rheinbay et al. 2013). H3K27me3 is a repressive mark prototypical of the polycomb repressive complex (Boyer et al. 2006; Zhou et al. 2011). Thus, the epigenetic pattern at the HOTAIR regulatory element in glioma cells and glioma stem cells suggests a possible bivalent function.

Development of high-order chromosome conformation capture techniques has facilitated analysis of long-range DNA interactions and enabled analysis of regulatory elements that enhance or repress gene expression through long-range interactions (Wei et al. 2013; Liu et al. 2017; Luo et al. 2017; Qian et al. 2019; Su et al. 2019). We hypothesized that the HOTAIR element may function in glioma progression or chemosensitivity. Here, we investigate the regulatory network governed by the element and discover

(c) 2020 Zhang et al. This article is distributed exclusively by Cold Spring Harbor Laboratory Press for the first six months after the full-issue publication date (see http://genome.cshlp.org/site/misc/terms.xhtml). After six months, it is available under a Creative Commons License (Attribution-NonCommercial 4.0 International), as described at http://creativecommons.org/licenses/ by-nc/4.0. 
A

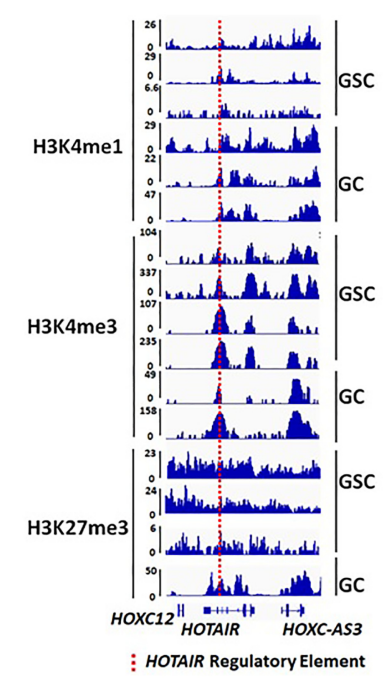

B

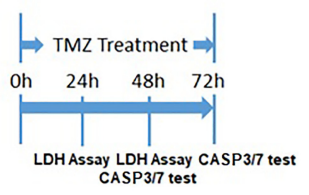

E

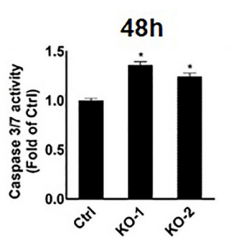

$\mathbf{F}$

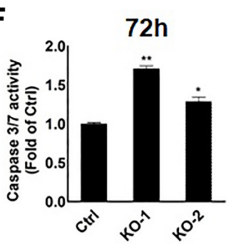

G

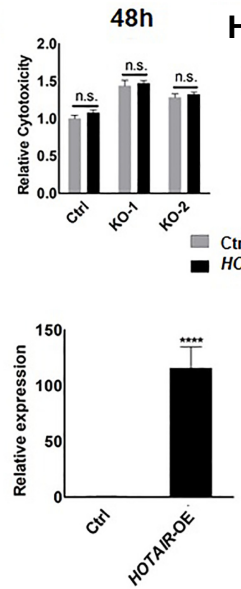

$72 \mathrm{~h}$
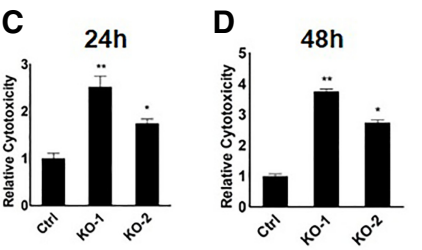

$\mathbf{J}$
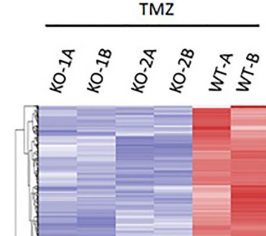

H
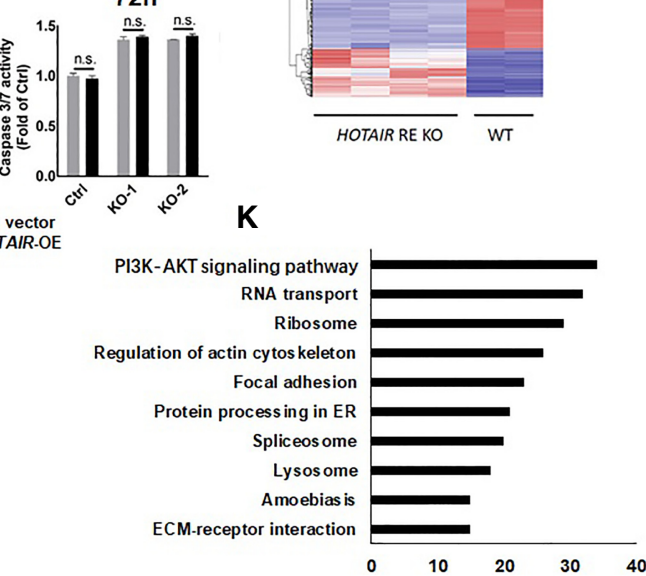

Figure 1. Deletion of the HOTAIR regulatory element increases glioma cells' sensitivity to TMZ. (A) $\mathrm{H} 3 \mathrm{~K} 4 \mathrm{me} 1, \mathrm{H} 3 \mathrm{~K} 4 \mathrm{me} 3, \mathrm{H} 3 \mathrm{~K} 27 \mathrm{me} 3 \mathrm{ChIP}$ of the region containing the HOTAIR regulatory element (red line) in glioma cells (GC) and glioma stem cells (GSC) derived from human tumor. (B) Schematic showing the timing of $\mathrm{LDH}$ and Caspase 3/7 assays. U251 glioma cells were treated with TMZ (1 mM) for 24-72 h. $(C, D)$ An LDH assay was used to detect LDH release in wild-type U251 cells (Ctrl) and HOTAIR regulatory element knockout $\mathrm{U} 251$ lines (KO-1, KO-2). LDH release was assayed after cells were treated $24 \mathrm{~h}$ and $48 \mathrm{~h}$ with TMZ $(1 \mathrm{mM})$. Data represent means \pm S.E.M. of three independent experiments. $\left(^{*}\right) P<0.05$, (**) $P<0.01$ compared with control. $(E, F)$ Proteolytic activities of caspase 3/7 were tested in wild-type U251 cells (Ctrl) and HOTAIR regulatory element knockout U251 cells (KO-1, $\mathrm{KO}-2)$ treated $48 \mathrm{~h}$ and $72 \mathrm{~h}$ with TMZ. Data represent means \pm S.E.M. of three independent experiments. $\left({ }^{*}\right) P<0.05,\left({ }^{* *}\right) P<0.01$ compared with control. (G) LDH assay was used to detect LDH release in wild-type U251 cells (Ctrl) and HOTAIR regulatory element knockout U251 lines (KO-1, KO-2) with HOTAIR overexpression (HOTAIR-OE) or transfected with control vector. LDH release was tested after cells were treated $48 \mathrm{~h}$ with TMZ. Data represent means \pm S.E.M. of three independent experiments. $(H)$ Proteolytic activities of caspase 3/7 were tested in wild-type U251 cells (Ctrl) and HOTAIR regulatory element knockout U251 cells (KO-1, KO-2) with HOTAIR overexpression (HOTAIR-OE) or transfected with control vector. Caspase 3/7 activities were tested after cells were treated $72 \mathrm{~h}$ with TMZ. Data represent means \pm S.E.M. of three independent experiments. (I) RT-qPCR analysis of HOTAIR in wild-type U251 cells transfected with control plasmid or HOTAIR overexpression plasmid. Data represent means \pm S.E.M. of three independent experiments. $\left.{ }^{* * * *}\right) P<0.0001$ compared with corresponding cells transfected with Ctrl vector. ( /) Comparison of gene expression in TMZ-treated (1 mM, $72 \mathrm{~h}$ ) HOTAIR regulatory knockout (KO) and wild-type (WT) cell lines. Heat map shows clustering of differentially expressed genes in $\mathrm{KO}$ and WT glioma cells. KO-1A and B, KO-2A and B, WT-A and B refer to two biological replicates, respectively. $(K)$ Function of genes $(Q-v a l u e<0.01)$ as analyzed by DAVID.

that the HOTAIR regulatory element modulates glioma cell TMZ sensitivity through long-range regulation of both CALCOCO1 and ZC3H1O.

\section{Results}

Deletion of the HOTAIR regulatory element increases glioma cell sensitivity to $\mathrm{TMZ}$

The HOTAIR regulatory element is marked by H3K4me1, H3K4me3, and H3K27me3 in glioma cells and glioblastoma stem cells derived from human tumors based on ChIP data reported by Rheinbay and colleagues (Fig. 1A; Rheinbay et al. 2013). To investigate whether the HOTAIR regulatory element functions in glioma cell sensitivity to TMZ, we used the CRISPR-Cas9 system to delete that element in U251 cells, a human GBM line. Two knockout lines (KO-1 and KO-2) were used in all of the following experiments (Supplemental Fig. S1A,B). We then treated both KO lines plus WT U251 cells with high $(1 \mathrm{mM})$ doses of TMZ to assess drug sensitivity, based on lactate dehydrogenase (LDH) release and caspase 3/7 activity. $\mathrm{LDH}$ is a stable cytosolic enzyme that is released upon cell lysis which indicates cell death induced by TMZ, while increased caspase $3 / 7$ activity indicates apoptosis. LDH release was evaluated $24 \mathrm{~h}$ and $48 \mathrm{~h}$ after TMZ treatment and Caspase 3/7 activities at $48 \mathrm{~h}$ and $72 \mathrm{~h}$ (Fig. 1B). Relative to WT U251 cells treated with TMZ, LDH release in $\mathrm{KO}-1$ and $\mathrm{KO}-2$ glioma cells was significantly increased approximately two- to threefold at $24 \mathrm{~h}$ and two- to fourfold at $48 \mathrm{~h}$ (Fig. 1C,D). Caspase 3/7 activities also increased markedly in TMZ-treated KO-1 and KO-2 glioma cells relative to $\mathrm{WT}$ U251 cells treated with TMZ at $48 \mathrm{~h}$ and $72 \mathrm{~h}$ (Fig. 1E,F). These data suggest that loss of the HOTAIR regulatory element increases glioma cells' sensitivity to TMZ. RT-qPCR analysis showed that HOTAIR mRNA is expressed at a low level in U251 cells (Supplemental Fig. S2A), and no significant change of HOTAIR's expression was detected in HOTAIR RE KO cells (Supplemental Fig. S2B). Moreover, overexpression of the HOTAIR lncRNA did not antagonize the increase in sensitivity to TMZ in either $\mathrm{KO}$ line (Fig. 1G,H): LDH release and caspase 3/ 7 activity were comparable in $\mathrm{KO}$ cells transduced with control vector and $\mathrm{KO}$ cells overexpressing HOTAIR following

\section{Genome Research}

www.genome.org 
TMZ treatment $(1 \mathrm{mM})$. U251 cells were transfected with HOTAIR overexpression plasmids, and overexpression efficiency was verified by RT-qPCR (Fig. 1I). RNA-seq analysis of control and KO U251 cells indicated global changes in mRNA levels in KO relative to WT cells treated with TMZ (1 mM, $72 \mathrm{~h}$ ) (Fig. 1J). Gene Ontology analysis of significantly changed genes $(Q$ value $<0.01)$ based on the DAVID knowledgebase (https://david.ncifcrf.gov) showed that the top pathway altered was PI3K-AKT signaling (Fig. 1K). Accordingly, the PI3K-AKT pathway is reportedly associated with TMZ resistance in glioma cells (Shi et al. 2017; Zhong et al. 2018).

\section{Long-range interactions of the HOTAIR regulatory element} with multiple target genes

Given that HOTAIR overexpression did not reverse increases in TMZ sensitivity in KO glioma cells, we surmised that the HOTAIR regulatory element itself has a complex function in regulatory networks in glioma cells. To search for target genes potentially regulated by this element, we performed Capture Hi-C in U251 glioma cells to analyze the interactome. To do so, we selected a 723-bp bait region (Fig. 2A) and performed Capture Hi-C based on a previously reported protocol (Davies et al. 2016; Belaghzal et al. 2017). Target sites from two biological replicates are shown in Circos plots (Krzywinski et al. 2009) in Figure 2, B and C, where red and blue lines, respectively, represent possible interactions in the two replicates. That analysis revealed that $67 \%$ and $63.2 \%$ are in cis interaction sites, respectively, in the two biological replicates. Mapping of these interaction sites to the human genome led to
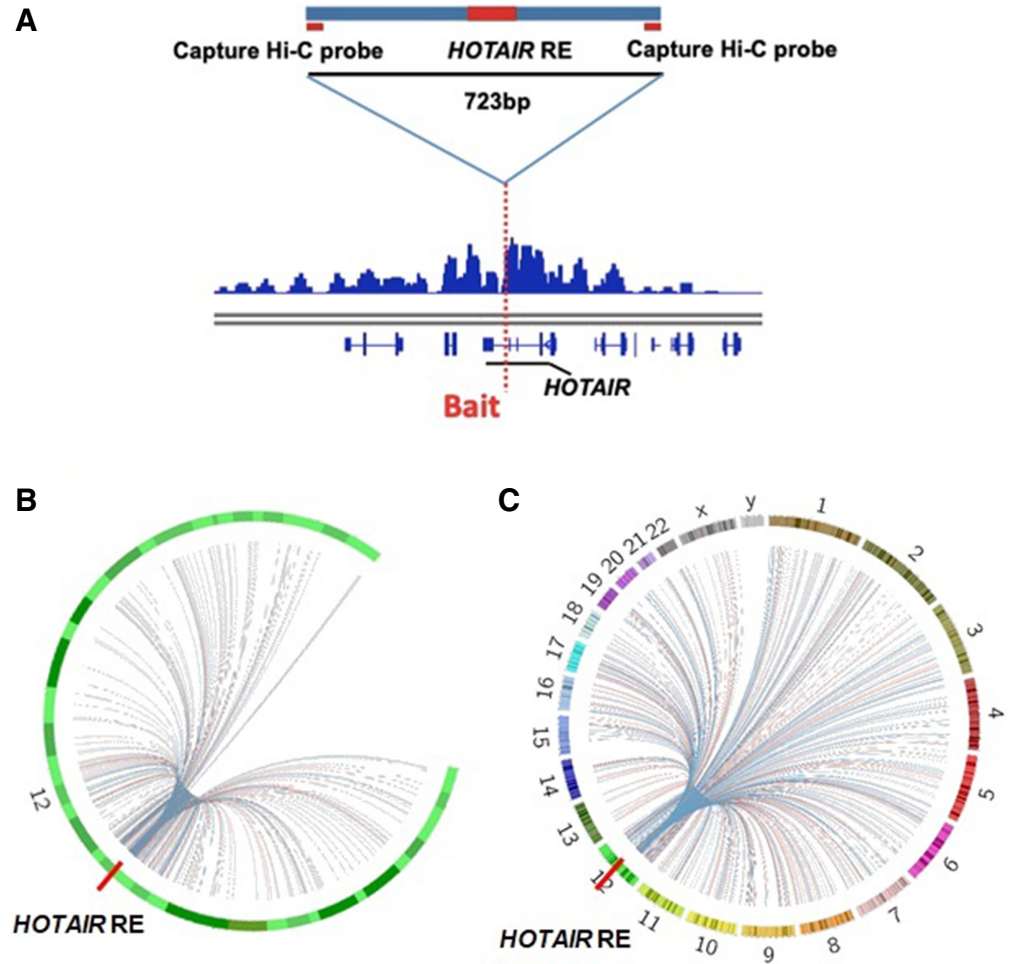

Figure 2. Intra- and inter-chromosomal interactions with the HOTAIR regulatory element. $(A)$ The 723-bp Capture $\mathrm{Hi}-\mathrm{C}$ bait region and Capture $\mathrm{Hi}-\mathrm{C}$ signal near the bait region. $(B)$ Circos plot showing genome-wide intra-interactions indicated by curves extending from the bait region; the two biological replicates are in red and blue, respectively. HOTAIR RE: HOTAIR regulatory element. (C) Circos plot showing genome-wide intra- and inter-interactions indicated by curves extending from the bait region; the two biological replicates are in red and blue, respectively. HOTAIR RE: HOTAIR regulatory element. identification of 89 target genes reproducible in both. These genes represent possible interactions with the HOTAIR regulatory element. Among the 89, 84 were located in cis with the element, and five were in trans.

To identify genes potentially regulated by the HOTAIR regulatory element, we performed RNA-seq on both wild-type and HOTAIR KO U251 cell lines (Fig. 3A). These genes may be direct or indirect targets of the regulatory fragment. When combined with Capture Hi-C, it may reveal genes that are directly regulated by the regulatory fragment. Positive candidate genes identified in both RNA-seq and Capture Hi-C are shown in Fig. 3B. Genes with significant changes in mRNA levels in enhancer $\mathrm{KO}$ relative to WT U251 cells (fold-change $>1.5$ or $<-1.5$; $Q$ value $<0.05$ ) are denoted by gene names (Fig. 3B). Overall, eight genes may be directly regulated by the HOTAIR regulatory element (Fig. 3B), including seven up-regulated in $\mathrm{KO}$ cells and one down-regulated.

Given that deletion of the HOTAIR regulatory element increased glioma cell sensitivity to TMZ, we asked which target genes function in this outcome. To do so, we first analyzed patient survival data associated with the eight target genes (Fig. 3C), which were available through The Cancer Genome Atlas (TCGA) database. Our analysis of survival curves indicated that three candidates (CALCOCO1, ZC3H1O, and TNS2) showed a pattern consistent with our in vitro data: All three were up-regulated in HOTAIR regulatory element $\mathrm{KO}$ cells, and accordingly, high levels of all three corresponded to longer survival time in glioma patients. These observations suggest overall that the HOTAIR regulatory element may function as a silencer of these candidate genes in glioma and that this activity is associated with TMZ sensitivity.

Long-range interactions between HOTAIR regulatory element and target genes are associated with $\mathrm{TMZ}$ sensitivity

We next assessed CALCOCO1, ZC3H1O, and TNS2 expression in WT U251 glioma cells and the two KO lines. RT-qPCR analysis showed increased CALCOCO1 and ZC3H1O transcript levels in $\mathrm{KO}$ lines relative to WT U251 cells with or without treatment of TMZ $(1 \mathrm{mM}, 72 \mathrm{~h})$, while TNS2 mRNA levels were comparable in both (Supplemental Fig. S3), suggesting that TNS2 is a false positive. In addition, TMZ treatment $(1 \mathrm{mM}, 72 \mathrm{~h})$ significantly up-regulated CALCOCO1 and ZC3H1O in both WT U251 cells and KO lines as shown by RT-qPCR (Fig. 4A) and western blot (Fig. 4B). Thus, we confined our analysis in the rest of the study to CALCOCO1 and ZC3H1O.

The CALCOCO1 and ZC3H1O loci are $\sim 0.25 \mathrm{Mb}$ and $\sim 2.2 \mathrm{Mb}$ distant from the HOTAIR regulatory element region, respectively. The interactions between the HOTAIR regulatory element and $C A L$ COCO1 or ZC3H1O genes, respectively, are shown by Capture Hi-C (Fig. 4C,D). A Chromatin Conformation Capture (3C) assay was then performed to further 
A

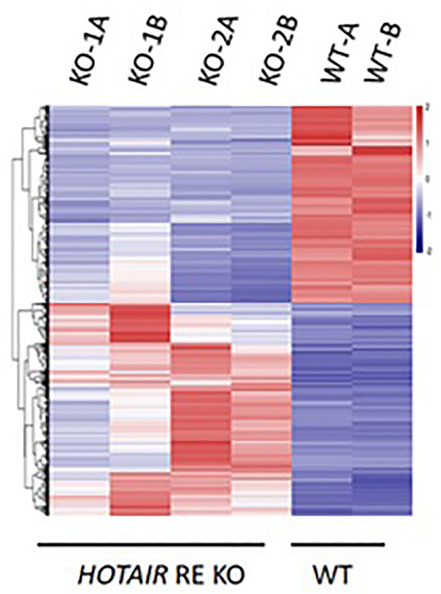

B

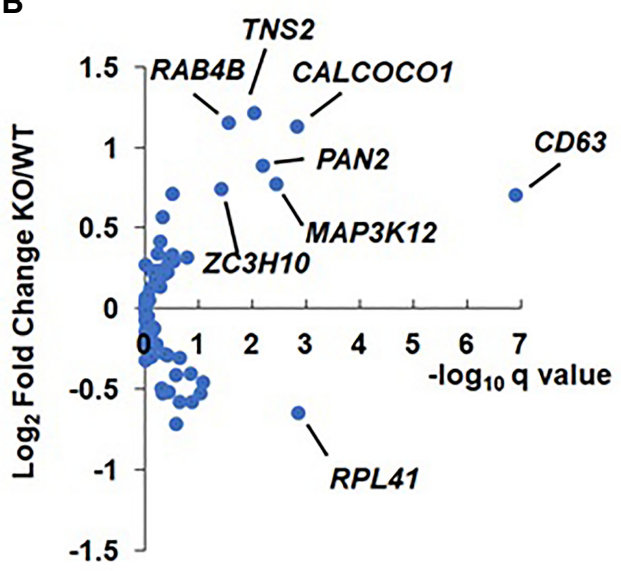

C

\begin{tabular}{|c|c|c|c|c|c|c|c|} 
& KOWT & & KOWT & & KOWT & & KOWT \\
\hline CALCOC01 & Up & ZC3H10 & Up & TNS2 & Up & RPL41 & DoWn
\end{tabular}
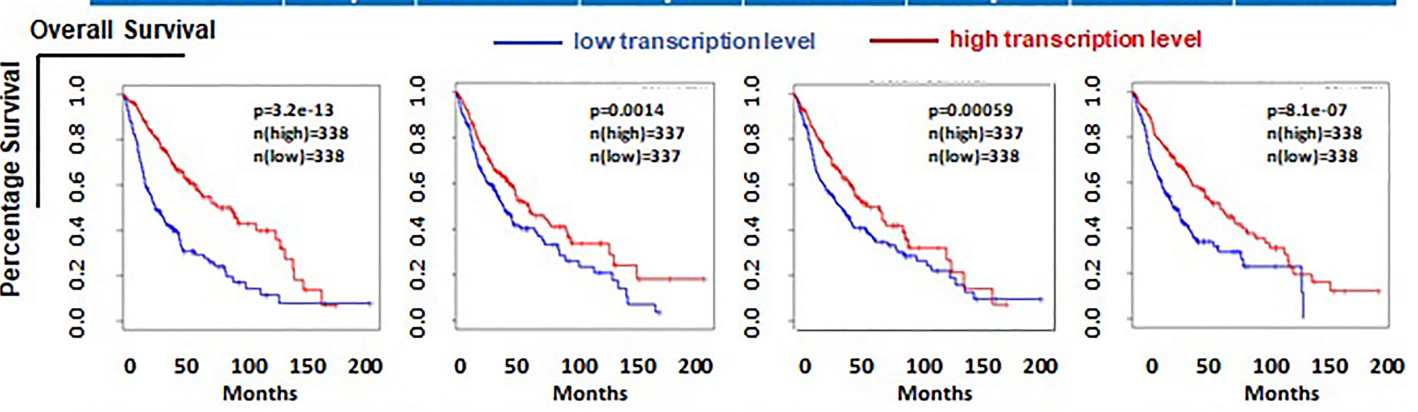

\begin{tabular}{|c|c|c|c|c|c|c|c|}
\hline & KOWT & & KONT & & KOWT & & KONT \\
\hline CD63 & Up & MAP3K12 & Up & PAN2 & Up & RAB4B & Up \\
\hline
\end{tabular}
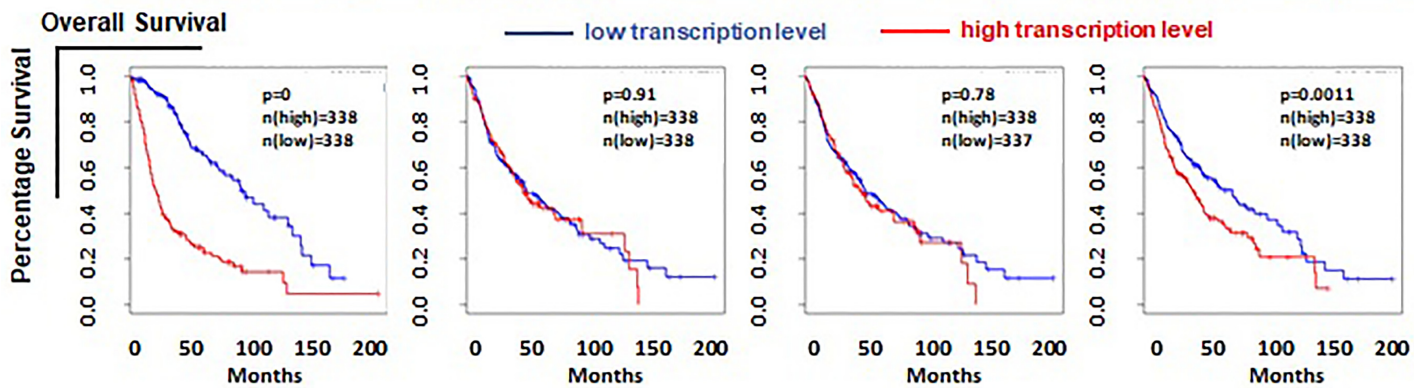

Figure 3. Potential target genes regulated by the HOTAIR regulatory element. ( $A$ ) Comparison of gene expression in HOTAIR regulatory knockout (KO) and wild-type (WT) glioma lines. Heat map shows clustering of differentially expressed genes in KO and WT glioma cells. KO-1A and B, KO-2A and B, WT-A and $B$ refer to two biological replicates, respectively. $(B)$ Comparison of fold-changes in gene expression based on RNA-seq data and corresponding Capture $\mathrm{Hi}-\mathrm{C}$ signal. Eighty-nine potential target genes reproducible in two biological replicates are shown on the plot. Among them, eight with significant expression change between KO and WT (adjusted $P$-value $<0.05$, and fold-change $>1.5$ or $<-1.5$ ) are marked with the gene name. (C) Shown are the eight target genes and associated survival data from glioma patients.

define these interactions (Fig. 4E,F). A C5 fragment at the CALCOCO1 promoter and $\mathrm{Z} 3$ fragment at the $\mathrm{ZC} 3 \mathrm{H} 10$ promoter showed significantly higher interaction frequency with the HOTAIR regulatory element region compared to the neighboring regions. The interaction frequencies between the HOTAIR regulatory element and C5 and Z3 fragments were significantly decreased in U251 cells treated with TMZ (Fig. 4E,F). In addition, we performed Hi-C based on a previously reported protocol (Belaghzal et al. 2017) to measure changes of interactions between the HOTAIR element and CALCOCO1 or ZC3H1O in U251 cells treated with TMZ ( $1 \mathrm{mM}, 72 \mathrm{~h})$. Analysis of Z-scores showed widespread changes in long-range interactions near the HOTAIR regulatory element in TMZ-treated U251 cells compared with U251 cells not treated with the drug and decreased interactions between the HOTAIR element and CALCOCO1 or ZC $3 H 10$ in U251 cells treated with TMZ (Supplemental Fig. S4). These data suggest that interactions between the HOTAIR regulatory element and CALCOCO1 and $\mathrm{ZC} 3 \mathrm{H} 10$ are associated with TMZ-induced cell death.

\section{Genome Research}

www.genome.org 
A

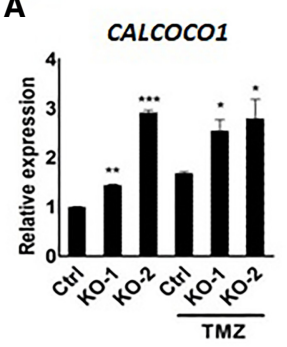

C

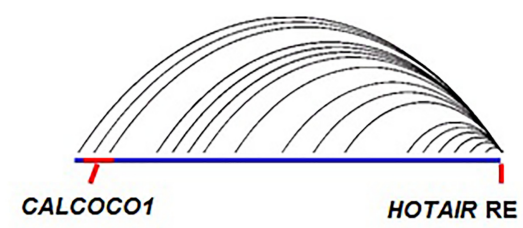

E
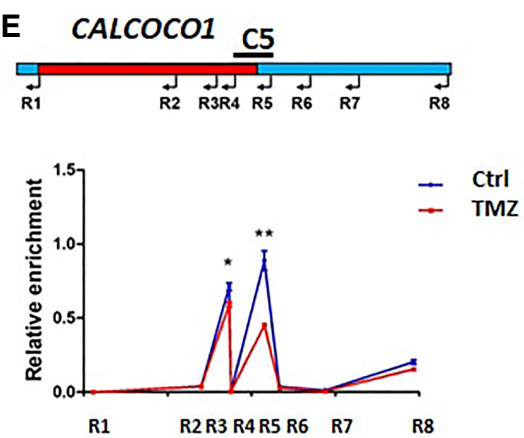

B

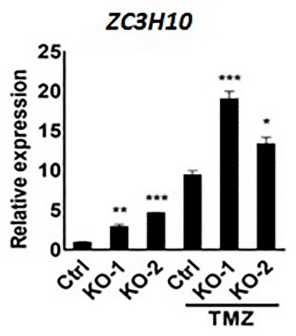

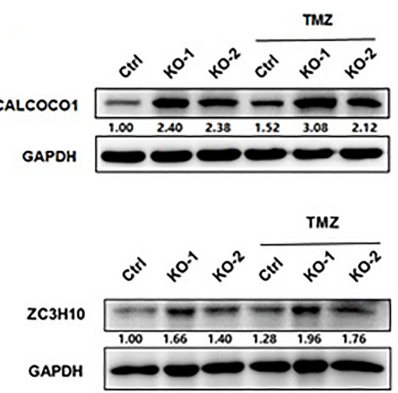

D

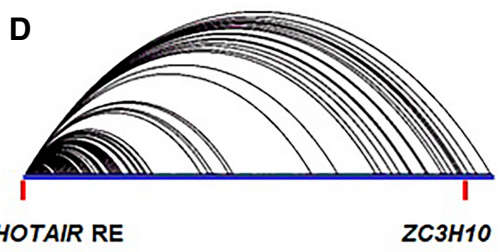

$\mathbf{F}$
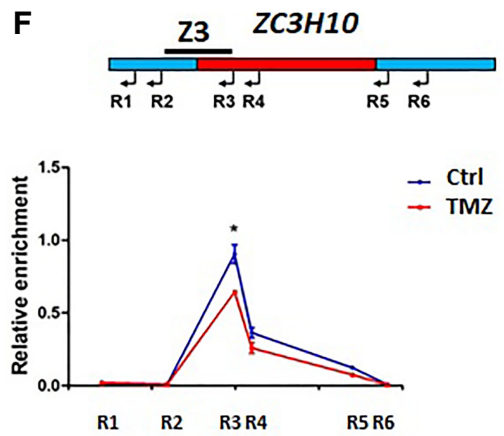

- ctrl

- TMZ

Figure 4. CALCOCO1 and $Z C 3 H 10$ are potentially regulated by the HOTAIR regulatory element. (A) RT-qPCR analysis of mRNA (independent mRNA library) levels of potential target genes CALCOCO1 and $Z C 3 H 10$. Data represent means \pm S.E.M. of three independent experiments. $(*) P<0.05,\left({ }^{* *}\right) P<$ $0.01,(* *) P<0.001$ compared with corresponding Ctrl cells. $(B)$ Western blot analysis of CALCOCO1 and $\mathrm{ZC} 3 \mathrm{H} 10$ in wild-type U251 cells (Ctrl) and HOTAIR regulatory element knockout U251 lines (KO-1, KO-2) that were treated with or without TMZ $(1 \mathrm{mM}, 72 \mathrm{~h})$. Relative protein levels were quantified by using Image). (C) Capture Hi-C loop view shows interactions between HOTAIR RE and CALCOCO1. (D) Capture Hi-C loop view shows interactions between HOTAIR RE and ZC3H10. (E) CALCOCO1HOTAIR RE interaction analyzed by $3 \mathrm{C}$ assay in U251 cells treated with or without TMZ (1 mM, $72 \mathrm{~h})$. Interaction frequency was calculated as described in the Methods. Data represent means \pm S.E.M. of three independent experiments. $\left(^{*}\right) P<0.05,\left({ }^{* *}\right) P<0.01$, compared with corresponding relative enrichment in U251 cells treated with TMZ. $(F)$ ZC3H10-HOTAIR RE interaction analyzed by $3 \mathrm{C}$ assay in U251 cells treated with or without TMZ $(1 \mathrm{mM}, 72 \mathrm{~h})$. Interaction frequency was calculated as described in the Methods. Data represent means \pm S.E.M. of three independent experiments. $\left.{ }^{*}\right) P<0.05$, compared with corresponding relative enrichment in U251 cells treated with TMZ.

CALCOCOI and ZC3H1O down-regulation decreases TMZ sensitivity in glioma cells lacking the HOTAIR regulatory element

We next asked whether CALCOCO1 or ZC3H1O regulation by the HOTAIR regulatory element modulates glioma cell sensitivity to TMZ. To do so, we undertook CALCOCO1 or ZC $3 H 10$ knockdown using siRNAs. RT-qPCR analysis of U251 cells and U251 cells transfected with siRNAs verified that CALCOCO1 or ZC3H1O siRNA transfection down-regulated CALCOCO1 or ZC3H10 0.3-fold or 0.4 -fold relative to the control, respectively (Fig. 5A-D). Transfection of cells with both CALCOCO1 and ZC $3 H 10$ siRNAs down-regulated CALCOCO1 and ZC3H10 $\sim 0.3$-fold or less relative to the control (Fig. 5E,F). When we knocked down CALCOCO1 or ZC3H1O separately in WT and KO lines, we observed no significant change in LDH release (Fig. 5G,H) or in caspase 3/7 activities (Fig. 5I,J) in sicontrol TMZ-treated $(1 \mathrm{mM}) \mathrm{KO}$ relative to TMZ-treated $(1 \mathrm{mM}) \mathrm{KO}$ cells transfected with CALCOCO1 or ZC3H1O siRNAs. However, combined knockdown of CALCOCO1 and ZC3H1O markedly decreased TMZ sensitivity in HOTAIR regulatory element $\mathrm{KO}$ glioma lines based on decreased $\mathrm{LDH}$ release and caspase 3/7 activities (Fig. 5K,L), suggesting that CALCOCO1 and ZC3H1O function synergistically to modulate TMZ sensitivity.

\section{Discussion}

Development of chromosome conformation capture technologies has facilitated study of long-range gene regulation by regulatory elements (Dekker et al. 2002; Dostie et al. 2006; Simonis et al. 2006; Lieberman-Aiden et al. 2009; Davies et al. 2016). Using those technologies, here, we show that deletion of a recently identified HOTAIR regulatory element in U251 glioma cells increased sensitivity of those cells to TMZ, and HOTAIR overexpression could not reverse this change in TMZ sensitivity. Thus, we suggest that the element modulates glioma cell TMZ sensitivity through long-range regulation of other target genes, based in part on our review of the pattern of epigenetic marks at this locus. Analysis of Capture Hi-C, RNA-seq, and patient survival data revealed that CALCOCO1 and $\mathrm{ZC} 3 \mathrm{H} 10$ may be key target genes regulated longrange by the HOTAIR element and that these genes regulate glioma cell sensitivity to TMZ.

Bivalent chromatin structure has been previously reported (Azuara et al. 2006; Bernstein et al. 2006; $\mathrm{Ku}$ et al. 2008; Vastenhouw and Schier 2012). Bernstein et al. proposed that bivalent chromatin domains marked by H3K4 methylation and H3K27 methylation silence some developmental genes in embryonic stem cells but keep these genes poised for activation (Bernstein et al. 2006). A similar function for bivalent chromatin has also been reported in cancer cells (Zaidi et al. 2017). Moreover, Zhang et al. identified the HOTAIR regulatory element as an enhancer in ESCC (Zhang et al. 2014), while our data suggest that this regulatory element silences $C A L C O C O 1$ and $Z C 3 H 1 O$ in glioma and also functions as an enhancer for other genes, like RPL41 (Supplemental Fig. S5). Our combining of Capture Hi-C with RNA-seq data revealed eight target genes potentially regulated by the HOTAIR element, seven up-regulated in cells lacking the element and one down-regulated, again suggesting a bivalent function in glioma cells. More investigations are needed to confirm this function. We focused on regulation of two of them, CALCOCO1 and ZC3H1O, as their expression patterns are consistent with patient survival data. Moreover, experiments in which we knocked out the regulatory 
A
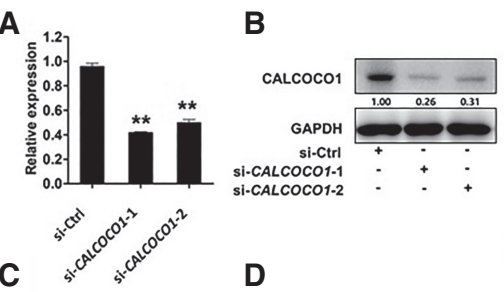

D

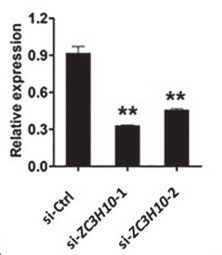

G

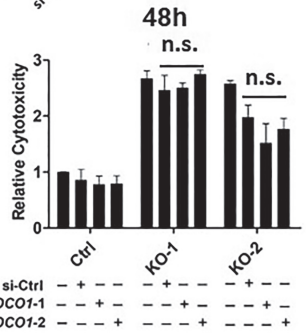

|

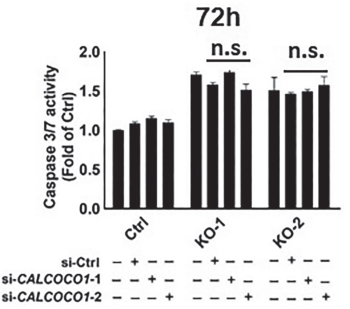

K

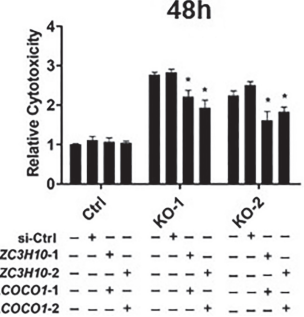

E

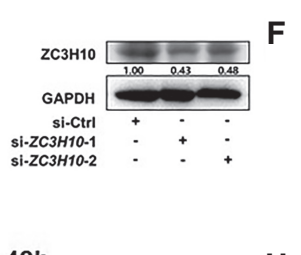

H

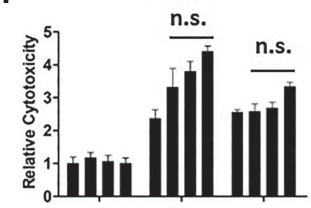

cs

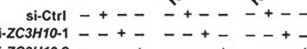

si-ZC3H10-1 --++--++--+-

J
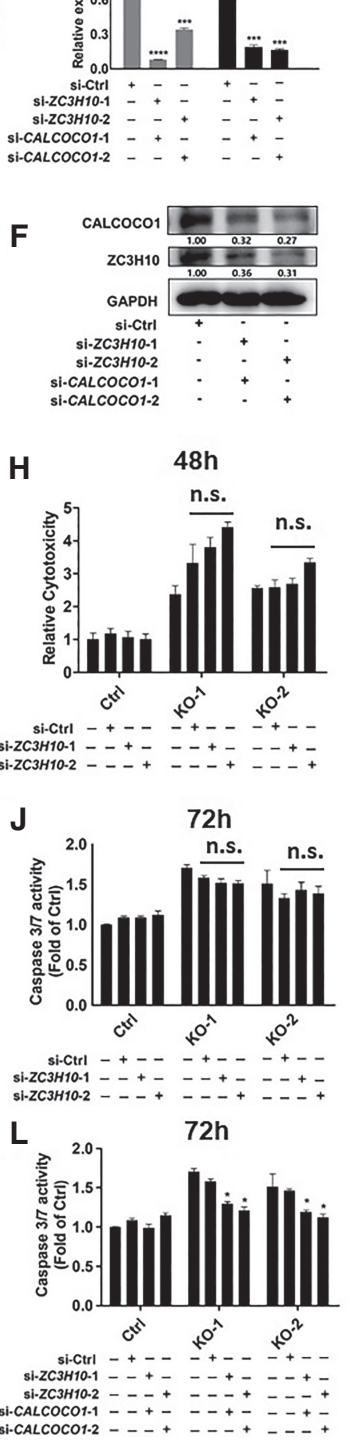
西

ZC3H10
CALCOCO1 
regulate DNA methylation, as TMZ is an alkylating chemotherapeutic agent (Wick et al. 2014). However, ours is one of the few studies to investigate this mechanism in view of long-range gene regulation networks. Our study reveals a new mechanism underlying TMZ sensitivity related to high-order chromatin structures in which the HOTAIR element modulates TMZ sensitivity through long-range regulation networks. Previous studies have reported methods to modify high-order chromatin structure as a means to change target gene expression (Deng et al. 2012, 2014; Morgan et al. 2017). Future analysis should focus on whether such approaches could represent an alternative method to improve prognosis of glioma patients.

In summary, our study shows that a recently identified HOTAIR regulatory element functions as a silencer to modulate glioma cell sensitivity to TMZ through long-range regulation of the targets CALCOCO1 and ZC $3 H 1 O$.

\section{Methods}

\section{Cell culture}

The GBM cell line U251 was obtained from the National Infrastructure of Cell Line Resource (China). U251 cells were incubated at $37^{\circ} \mathrm{C}$ with $5 \% \mathrm{CO}_{2}$ and cultured in Dulbecco's Modified Eagle Medium (GIBCO), supplemented with $10 \%$ fetal bovine serum (FBS) (BI) and 1\% penicillin-streptomycin (GIBCO). All cells were tested for mycoplasma contamination each month. All cells used in this paper were mycoplasma-free.

\section{CRISPR-Cas9-mediated deletions}

U251 glioma cells were transfected with Cas9 and guide RNA plasmids that target the HOTAIR regulatory element region (hg19_Chr 12: 54,360,188-54,360,822). Guide RNAs were designed using the MIT CRISPR Design website (https://zlab.bio/guide-designresources). To minimize potential off-target effects of guide RNA, only high-score guide RNAs (score $>85$ ) were used. Guide RNA sequences are listed in Supplemental Table S1. Cell clones were genotyped by PCR, and two clones with homozygous deletion of the HOTAIR regulatory element were used for experiments. Genotyping PCR primers are listed in Supplemental Table S1. GRCh37 (hg19) was used in this study. The differences between GRCh37 and GRCh38 did not affect the deletion region of the regulatory element and the relative position of the regulatory element and its targets; therefore, using GRCh38 would not significantly affect the conclusions in this study.

\section{Lactate dehydrogenase assay}

Lactate dehydrogenase in the culture medium was evaluated using the LDH cytotoxicity kit (Promega). In brief, cells were cultured in 96-well plates. After cells were treated with TMZ or transfected with siRNA or overexpression plasmids, the culture medium was collected and LDH activity was assayed in accordance with the manufacturer's instructions. The OD value at $490 \mathrm{~nm}$ was measured using a microplate reader. Levels of released LDH of each group were calculated as a percentage of the total amount (positive control as described in the kit protocol).

\section{Caspase $3 / 7$ activity measurements}

A Caspase-Glo 3/7 kit (Promega) was used to evaluate caspase 3/7 activities in U251 cells based on the manufacturer's instruction. In brief, cells were harvested and incubated with $100 \mu \mathrm{L}$ Caspase-Glo $3 / 7$ reagent for $1 \mathrm{~h}$ on a rotary shaker in the dark. Luminescence of each sample was measured at 485/530 nm using a microplate reader. Relative caspase 3/7 activity was calculated to evaluate foldchanges of samples treated with TMZ or transfected with siRNA or overexpression plasmids relative to controls.

\section{HOTAIR overexpression}

HOTAIR was amplified by PCR with NEBNext High-Fidelity $2 \times$ PCR Master Mix (New England BioLabs) and subsequently cloned into the pCDH-CMV-MCS-EF1-Puro overexpression plasmid (System Biosciences) using restriction enzymes NheI (New England BioLabs) and NotI (New England BioLabs). Plasmids were amplified in One Shot Stbl3 chemically competent E. coli (Thermo Fisher Scientific) with ampicillin (Bio Basic Canada, Inc.) selection. Plasmids were transiently transfected using Lipofectamine 3000 (Thermo Fisher Scientific) according to the manufacturer's instructions.

\section{RNA-seq}

Total RNA was extracted from U251 glioma cells or HOTAIR regulatory element KO U251 cells treated with or without TMZ (1 mM, $72 \mathrm{~h}$ ). Barcoded RNA-seq libraries were sequenced as 150-bp paired-end reads using the Illumina HiSeq 4000 platform. Reads were mapped to the human reference genome (hg19) using HISAT (Kim et al. 2015) with a GENCODE GTF file supplied as gene model annotations. HTSeq (Anders et al. 2015) was used to quantitate transcript abundance for each gene. DESeq2 (Love et al. 2014) was used to perform normalization and regularized log transformations on read counts.

\section{Survival curve}

Clinical glioma patients' survival data were obtained from TCGA. Patient samples were divided into two groups based on the expression level of specific genes indicated in the main text. Samples with gene expression levels higher or lower than the median were marked as "high expression group" or "low expression group," respectively. $P$-value $<0.0001$ indicates a significant survival difference between the two groups.

\section{Capture $\mathrm{Hi}-\mathrm{C}$ and $\mathrm{Hi}-\mathrm{C}$}

Capture $\mathrm{Hi}-\mathrm{C}$ and $\mathrm{Hi}-\mathrm{C}$ were performed as previously described (Davies et al. 2016; Belaghzal et al. 2017). The Hi-C library was generated with DpnII (New England BioLabs) digestion. Two biological replicates were assessed with $1 \times 10^{7} \mathrm{U} 251$ cells. The HiC library was then sheared to 200- to 300-bp fragments by sonication, and the library or precapture library was prepared using the NEBNext DNA library kit according to the manufacturer's instructions (New England BioLabs). Biotinylated probes and streptavidin beads (Invitrogen) were used to enrich the "bait" region and its linked chromatin fragments by two rounds of hybridization-capture approach. Capture Hi-C libraries were sequenced as 150-bp paired-end reads using the Illumina HiSeq $\mathrm{X}$ Ten platform. Capture Hi-C and Hi-C data were analyzed based on a pipeline proposed in previous studies (Supplemental Table S2; Servant et al. 2015; Davies et al. 2016; Belaghzal et al. 2017).

Two biotinylated DNA oligonucleotides were designed for both ends of the region containing the HOTAIR regulatory element, with the following sequence $\left(5^{\prime}-3^{\prime}\right)$ :

Biotin-

GATCGAAAGATAAAAAGAGGCGGGAGGGAGGCAGAAGC AAGGGTCGGCTGTTGCTCTCCCTCGCCCGGGCGCCTGC AGCAGTCTGGGTGGGCGGGGGCGCCATGACAAAGTGAA GGTCAA 
Biotin-

GTCTGAATGTTACGGTTTCCTTCAGAAAACAAGGCGGTA CACTCGGTAAGGAGAAGTGAGGCAGCAGGGGTGGGGA GGGCTGGTGCGGTAGGAGGCTTGGTTTTATTTCTTTCTC AGATC

\section{Quantitative real-time PCR}

Total RNA was extracted using TRIzol Reagent (Invitrogen) following the manufacturer's protocol. Five micrograms total RNA was reverse-transcribed into cDNA using a PrimeScript RT reagent kit (Takara). PCR analysis was performed using an SYBR Premix Ex Taq kit (Takara). Primer sequences used in the study are listed in Supplemental Table S3. GAPDH served as an internal reference, and the relative expression of messenger RNAs (mRNAs) was quantified using the $2^{-\Delta \Delta \mathrm{Ct}}$ method.

\section{Chomatin Conformation Capture (3C) assay}

The $3 \mathrm{C}$ assay was performed as previously described (Hagège et al. 2007). In brief, U251 cells were cross-linked with $1 \%$ formaldehyde and quenched with $125 \mathrm{mM}$ glycine. The $3 \mathrm{C}$ library was generated with HindIII (New England BioLabs) digestion and followed by ligation with T4 ligase (New England BioLabs). The ligated products were quantified by RT-qPCR. Primers used in the 3C assay are listed in Supplemental Table S4. Control 3C template was generated by using bacterial artificial chromosomes (BACs) RP11-657H18 and RP11-166L22 and DNA fragments that contain the HOTAIR regulatory element region, $C A L C O C O 1$ loci, and $Z C 3 H 1 O$ loci, respectively. BACs and DNA fragments were digested with HindIII and ligated. The ligated product from the control was used for normalization. The relative interaction frequency was calculated as $2^{\mathrm{Ct}(\mathrm{Control})-\mathrm{Ct}(3 \mathrm{C})}$

\section{Western blots}

Proteins were collected from glioma cells using RIPA lysis buffer with $1 \times$ protease inhibitor cocktail (Roche). Sample loading was based on the results of a Bicinchoninic Acid (BCA) assay. Proteins were separated by sodium dodecyl sulfate-polyacrylamide gel electrophoresis (SDS-PAGE), and then transferred to polyvinylidene difluoride (PVDF) $0.45-\mu \mathrm{m}$ membranes (Millipore). The membranes were blocked and incubated with primary antibody overnight at $4^{\circ} \mathrm{C}$, followed by secondary antibodies for $1 \mathrm{~h}$ at room temperature. Then, the blot bands were detected by Image Quant LAS 4000 with an Enhanced Chemiluminescence kit (Thermo Fisher Scientific). Relative protein levels were quantified by using ImageJ. Antibodies used in western blots were as follows: CALCOCO1 (Bioworld BS71035), ZC3H10 (Abcam ab127693), GAPDH (Santa Cruz Biotechnology sc-365062), goat anti-rabbit IgG-HRP (Sungene Biotech LK2001), and rabbit anti-mouse IgG-HRP (Abcam ab6728).

\section{siRNA transfection}

Glioma cells were transfected with siRNAs (Supplemental Table S5) using Lipofectamine 3000 reagent (Thermo Fisher Scientific). siRNA knockdown efficiency was verified by RT-qPCR $48-72 \mathrm{~h}$ later.

\section{Statistical analysis}

Data represent means \pm S.E.M.; statistical analysis was performed using Student's $t$-test. (*) $P<0.05,\left({ }^{* *}\right) P<0.01,\left(^{* *}\right) P<0.001$, $(* * *) P<0.0001$.

\section{Data access}

All sequencing data generated in this study have been submitted to the NCBI Gene Expression Omnibus (GEO; https://www.ncbi .nlm.nih.gov/geo/) under accession numbers GSE125629 and GSE125243.

\section{Competing interest statement}

The authors declare no competing interests.

\section{Acknowledgments}

This work was supported by the National Natural Science Foundation of China (Grant No. 31530027, 31701129, 81772687), National Key R\&D Program of China (Grant No. 2017YFA0102600), and the Natural Science Foundation of Tianjin City of China (Grant No. 18JCQNJC10100). We thank Dr. Lingyi Chen at the College of Life Sciences of Nankai University for providing the Cas9 plasmids. We thank Dr. Mulin Jun Li at the School of Basic Medical Sciences of Tianjin Medical University for his suggestions. Author contributions: L.Z., A.H., B.C., J.B., J.C., D.G., Y.Q., W.W., T.S., Z.Z., J.S., W.A., and F.A. conducted the experiments; L.Z. and W.L. designed the experiments; and L.Z. and W.L. wrote the paper.

\section{References}

Anders S, Pyl PT, Huber W. 2015. HTSeq-a Python framework to work with high-throughput sequencing data. Bioinformatics 31: 166-169. doi:10 .1093/bioinformatics/btu638

Azuara V, Perry P, Sauer S, Spivakov M, Jørgensen HF, John RM, Gouti M, Casanova M, Warnes G, Merkenschlager M, et al. 2006. Chromatin signatures of pluripotent cell lines. Nat Cell Biol 8: 532-538. doi:10.1038/ ncb1403

Belaghzal H, Dekker J, Gibcus JH. 2017. Hi-C 2.0: an optimized Hi-C procedure for high-resolution genome-wide mapping of chromosome conformation. Methods 123: 56-65. doi:10.1016/j.ymeth.2017.04.004

Bernstein BE, Mikkelsen TS, Xie X, Kamal M, Huebert DJ, Cuff J, Fry B, Meissner A, Wernig M, Plath K, et al. 2006. A bivalent chromatin structure marks key developmental genes in embryonic stem cells. Cell 125: 315-326. doi:10.1016/j.cell.2006.02.041

Boyer LA, Plath K, Zeitlinger J, Brambrink T, Medeiros LA, Lee TI, Levine SS, Wernig M, Tajonar A, Ray MK, et al. 2006. Polycomb complexes repress developmental regulators in murine embryonic stem cells. Nature 441: 349-353. doi:10.1038/nature04733

Clarke MJ, Mulligan EA, Grogan PT, Mladek AC, Carlson BL, Schroeder MA Curtin NJ, Lou Z, Decker PA, Wu W, et al. 2009. Effective sensitization of temozolomide by ABT-888 is lost with development of temozolomide resistance in glioblastoma xenograft lines. Mol Cancer Ther 8: 407-414. doi:10.1158/1535-7163.MCT-08-0854

Davies JO, Telenius JM, McGowan SJ, Roberts NA, Taylor S, Higgs DR, Hughes JR. 2016. Multiplexed analysis of chromosome conformation at vastly improved sensitivity. Nat Methods 13: 74-80. doi:10.1038/ nmeth.3664

Dekker J, Rippe K, Dekker M, Kleckner N. 2002. Capturing chromosome conformation. Science 295: 1306-1311. doi:10.1126/science.1067799

Deng W, Lee J, Wang H, Miller J, Reik A, Gregory PD, Dean A, Blobel GA. 2012. Controlling long-range genomic interactions at a native locus by targeted tethering of a looping factor. Cell 149: 1233-1244. doi:10 .1016/j.cell.2012.03.051

Deng W, Rupon JW, Krivega I, Breda L, Motta I, Jahn KS, Reik A, Gregory PD, Rivella S, Dean A, et al. 2014. Reactivation of developmentally silenced globin genes by forced chromatin looping. Cell 158: 849-860. doi:10 .1016/j.cell.2014.05.050

Dostie J, Richmond TA, Arnaout RA, Selzer RR, Lee WL, Honan TA, Rubio ED, Krumm A, Lamb J, Nusbaum C, et al. 2006. Chromosome Conformation Capture Carbon Copy (5C): a massively parallel solution for mapping interactions between genomic elements. Genome Res 16: 1299-1309. doi:10.1101/gr.5571506

Guardiola-Serrano F, Haendeler J, Lukosz M, Sturm K, Melchner $\mathrm{H}$ Altschmied J. 2008. Gene trapping identifies a putative tumor 
suppressor and a new inducer of cell migration. Biochem Biophys Res Commun 376: 748-752. doi:10.1016/j.bbrc.2008.09.070

Hagège $H$, Klous P, Braem C, Splinter E, Dekker J, Cathala G, de Laat W, Forné T. 2007. Quantitative analysis of chromosome conformation capture assays (3C-qPCR). Nat Protoc 2: 1722-1733. doi:10.1038/nprot .2007 .243

Kim JH, Li H, Stallcup MR. 2003. CoCoA, a nuclear receptor coactivator which acts through an N-terminal activation domain of p160 coactivators. Mol Cell 12: 1537-1549. doi:10.1016/S1097-2765(03)00450-7

Kim JH, Yang CK, Heo K, Roeder RG, An W, Stallcup MR. 2008. CCAR1, a key regulator of mediator complex recruitment to nuclear receptor transcription complexes. Mol Cell 31: 510-519. doi:10.1016/j.molcel.2008 .08 .001

Kim D, Langmead B, Salzberg SL. 2015. HISAT: a fast spliced aligner with low memory requirements. Nat Methods 12: 357-360. doi:10.1038/nmeth .3317

Kitange GJ, Carlson BL, Schroeder MA, Grogan PT, Lamont JD, Decker PA Wu W, James CD, Sarkaria JN. 2009. Induction of MGMT expression is associated with temozolomide resistance in glioblastoma xenografts. Neuro-Oncology 11: 281-291. doi:10.1215/15228517-2008-090

Krzywinski M, Schein J, Birol I, Connors J, Gascoyne R, Horsman D, Jones SJ Marra MA. 2009. Circos: an information aesthetic for comparative genomics. Genome Res 19: 1639-1645. doi:10.1101/gr.092759.109

Ku M, Koche RP, Rheinbay E, Mendenhall EM, Endoh M, Mikkelsen TS, Presser A, Nusbaum C, Xie X, Chi AS, et al. 2008. Genomewide analysis of PRC1 and PRC2 occupancy identifies two classes of bivalent domains. PLoS Genet 4: e1000242. doi:10.1371/journal.pgen.1000242

Lieberman-Aiden E, van Berkum NL, Williams L, Imakaev M, Ragoczy T, Telling A, Amit I, Lajoie BR, Sabo PJ, Dorschner MO, et al. 2009. Comprehensive mapping of long-range interactions reveals folding principles of the human genome. Science 326: 289-293. doi:10.1126/sci ence.1181369

Liu NQ, Ter Huurne M, Nguyen LN, Peng T, Wang SY, Studd JB, Joshi O, Ongen H, Bramsen JB, Yan J, et al. 2017. The non-coding variant rs1800734 enhances DCLK3 expression through long-range interaction and promotes colorectal cancer progression. Nat Commun 8: 14418. doi:10.1038/ncomms 14418

Love MI, Huber W, Anders S. 2014. Moderated estimation of fold change and dispersion for RNA-seq data with DESeq2. Genome Biol 15: 550. doi:10.1186/s13059-014-0550-8

Luo Z, Rhie SK, Lay FD, Farnham PJ. 2017. A prostate cancer risk element functions as a repressive loop that regulates HOXA13. Cell Rep 21: 1411-1417. doi:10.1016/j.celrep.2017.10.048

Morgan SL, Mariano NC, Bermudez A, Arruda NL, Wu F, Luo Y, Shankar G, Jia L, Chen $\mathrm{H}, \mathrm{Hu}$ JF, et al. 2017. Manipulation of nuclear architecture through CRISPR-mediated chromosomal looping. Nat Commun 8: 15993. doi:10.1038/ncomms15993

Pan W, Wu C, Su Z, Duan Z, Li L, Mi F, Li C. 2017. Genetic polymorphisms of non-coding RNAs associated with increased head and neck cancer susceptibility: a systematic review and meta-analysis. Oncotarget 8: 62508-62523. doi:10.18632/oncotarget.20096

Qian Y, Zhang L, Cai M, Li H, Xu H, Yang H, Zhao Z, Rhie SK, Farnham PJ, Shi J, et al. 2019. The prostate cancer risk variant rs55958994 regulates multiple gene expression through extreme long-range chromatin interaction to control tumor progression. Sci Adv 5: eaaw6710. doi:10.1126/ sciadv.aaw6710

Quinn JA, Jiang SX, Reardon DA, Desjardins A, Vredenburgh JJ, Friedman AH, Sampson JH, McLendon RE, Herndon JE II, Friedman HS. 2009. Phase II trial of temozolomide (TMZ) plus irinotecan (CPT-11) in adults with newly diagnosed glioblastoma multiforme before radiotherapy. J Neurooncol 95: 393-400. doi:10.1007/s11060-009-9937-x

Reifenberger G, Wirsching HG, Knobbe-Thomsen CB, Weller M. 2017. Advances in the molecular genetics of gliomas-implications for classification and therapy. Nat Rev Clin Oncol 14: 434-452. doi:10.1038/nrcli nonc. 2016.204

Rheinbay E, Suvà ML, Gillespie SM, Wakimoto H, Patel AP, Shahid M, Oksuz O, Rabkin SD, Martuza RL, Rivera MN, et al. 2013. An aberrant transcription factor network essential for Wnt signaling and stem cell maintenance in glioblastoma. Cell Rep 3: 1567-1579. doi:10.1016/j.celrep .2013.04.021

Sandmann T, Bourgon R, Garcia J, Li C, Cloughesy T, Chinot OL, Wick W, Nishikawa R, Mason W, Henriksson R, et al. 2015. Patients with proneural glioblastoma may derive overall survival benefit from the addition of bevacizumab to first-line radiotherapy and temozolomide: retrospective analysis of the AVAglio trial. J Clin Oncol 33: 2735-2744. doi:10.1200/ JCO.2015.61.5005

Schäfer A, Teufel J, Ringel F, Bettstetter M, Hoepner I, Rasper M, Gempt J, Koeritzer J, Schmidt-Graf F, Meyer B, et al. 2012. Aldehyde dehydrogenase $1 \mathrm{~A} 1-$ a new mediator of resistance to temozolomide in glioblastoma. Neuro-Oncology 14: 1452-1464. doi:10.1093/neuonc/nos270
Servant N, Varoquaux N, Lajoie BR, Viara E, Chen CJ, Vert JP, Heard E, Dekker J, Barillot E. 2015. HiC-Pro: an optimized and flexible pipeline for Hi-C data processing. Genome Biol 16: 259. doi:10.1186/s13059015-0831-X

Shi F, Guo H, Zhang R, Liu H, Wu L, Wu Q, Liu J, Liu T, Zhang Q. 2017. The PI3K inhibitor GDC-0941 enhances radiosensitization and reduces chemoresistance to temozolomide in GBM cell lines. Neuroscience 346: 298-308. doi:10.1016/j.neuroscience.2017.01.032

Simonis M, Klous P, Splinter E, Moshkin Y, Willemsen R, de Wit E, van Steensel B, de Laat W. 2006. Nuclear organization of active and inactive chromatin domains uncovered by chromosome conformation captureon-chip (4C). Nat Genet 38: 1348-1354. doi:10.1038/ng1896

Su G, Guo D, Chen J, Liu M, Zheng J, Wang W, Zhao X, Yin Q, Zhang L, Zhao $Z$, et al. 2019. A distal enhancer maintaining Hoxa1 expression orchestrates retinoic acid-induced early ESCs differentiation. Nucleic Acids Res 47: 6737-6752. doi:10.1093/nar/gkz482

Sun S, Lee D, Ho AS, Pu JK, Zhang XQ, Lee NP, Day PJ, Lui WM, Fung CF, Leung GK. 2013. Inhibition of prolyl 4-hydroxylase, $\beta$ polypeptide $(\mathrm{P} 4 \mathrm{HB})$ attenuates temozolomide resistance in malignant glioma via the endoplasmic reticulum stress response (ERSR) pathways. NeuroOncology 15: 562-577. doi:10.1093/neuonc/not005

van 't Veer LJ, Dai H, van de Vijver MJ, He YD, Hart AA, Mao M, Peterse HL, van der Kooy K, Marton MJ, Witteveen AT, et al. 2002. Gene expression profiling predicts clinical outcome of breast cancer. Nature 415: 530-536. doi:10.1038/415530a

Vastenhouw NL, Schier AF. 2012. Bivalent histone modifications in early embryogenesis. Curr Opin Cell Biol 24: 374-386. doi:10.1016/j.ceb 2012.03.009

Wang C, Li Y, Li YW, Zhang HB, Gong H, Yuan Y, Li WT, Liu HY, Chen J. 2018. HOTAIR lncRNA SNPs rs920778 and rs1899663 are associated with smoking, male gender, and squamous cell carcinoma in a Chinese lung cancer population. Acta Pharmacol Sin 39: 1797-1803. doi:10.1038/s41401-018-0083-x

Warren KE, Gururangan S, Geyer JR, McLendon RE, Poussaint TY, Wallace D, Balis FM, Berg SL, Packer RJ, Goldman S, et al. 2012. A phase II study of O6-benzylguanine and temozolomide in pediatric patients with recurrent or progressive high-grade gliomas and brainstem gliomas: a Pediatric Brain Tumor Consortium study. J Neurooncol 106: 643-649. doi:10.1007/s11060-011-0709-z

Wei Z, Gao F, Kim S, Yang H, Lyu J, An W, Wang K, Lu W. 2013. Klf4 organizes long-range chromosomal interactions with the Oct4 locus in reprogramming and pluripotency. Cell Stem Cell 13: 36-47. doi:10.1016/j stem.2013.05.010

Weller M, Wick W, Aldape K, Brada M, Berger M, Pfister SM, Nishikawa R, Rosenthal M, Wen PY, Stupp R, et al. 2015. Glioma. Nature Rev Dis Prim 1: 15017. doi:10.1038/nrdp.2015.17

Weng SL, Wu WJ, Hsiao YH, Yang SF, Hsu CF, Wang PH. 2018. Significant association of long non-coding RNAs HOTAIR genetic polymorphisms with cancer recurrence and patient survival in patients with uterine cervical cancer. Int J Med Sci 15: 1312-1319. doi:10.7150/ijms.27505

Wick W, Platten M, Meisner C, Felsberg J, Tabatabai G, Simon M, Nikkhah G, Papsdorf K, Steinbach JP, Sabel M, et al. 2012. Temozolomide chemotherapy alone versus radiotherapy alone for malignant astrocytoma in the elderly: the NOA-08 randomised, phase 3 trial. Lancet Oncol 13: 707-715. doi:10.1016/S1470-2045(12)70164-X

Wick W, Weller M, van den Bent M, Sanson M, Weiler M, von Deimling A Plass C, Hegi M, Platten M, Reifenberger G. 2014. MGMT testing-the challenges for biomarker-based glioma treatment. Nat Rev Neurol 10: 372-385. doi:10.1038/nrneurol.2014.100

Zaidi SK, Frietze SE, Gordon JA, Heath JL, Messier T, Hong D, Boyd JR, Kang M, Imbalzano AN, Lian JB, et al. 2017. Bivalent epigenetic control of oncofetal gene expression in cancer. Mol Cell Biol 37. doi:10.1128/ MCB.00352-17

Zhang X, Zhou L, Fu G, Sun F, Shi J, Wei J, Lu C, Zhou C, Yuan Q, Yang M. 2014. The identification of an ESCC susceptibility SNP rs920778 that regulates the expression of IncRNA HOTAIR via a novel intronic enhancer. Carcinogenesis 35: 2062-2067. doi:10.1093/carcin/bgu103

Zhong C, Chen Y, Tao B, Peng L, Peng T, Yang X, Xia X, Chen L. 2018. LIM and $\mathrm{SH} 3$ protein 1 regulates cell growth and chemosensitivity of human glioblastoma via the PI3K/AKT pathway. BMC Cancer 18: 722. doi:10 .1186/s12885-018-4649-2

Zhou VW, Goren A, Bernstein BE. 2011. Charting histone modifications and the functional organization of mammalian genomes. Nat Rev Genet 12: 7-18. doi:10.1038/nrg2905

Received March 30, 2019; accepted in revised form January 8, 2020. 


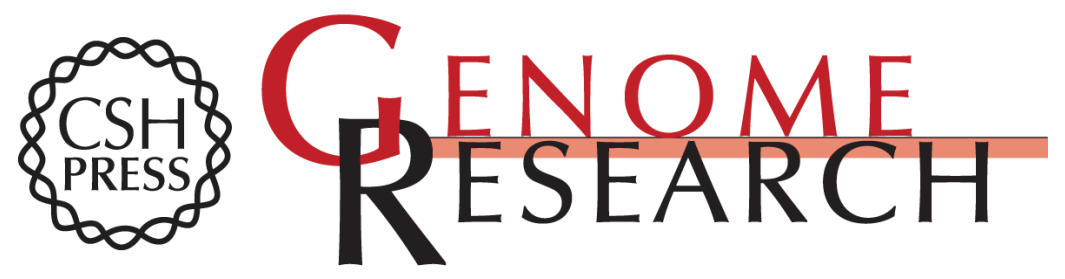

\section{A HOTAIR regulatory element modulates glioma cell sensitivity to temozolomide through long-range regulation of multiple target genes}

Lei Zhang, Anshun He, Bohan Chen, et al.

Genome Res. 2020 30: 155-163 originally published online January 17, 2020

Access the most recent version at doi:10.1101/gr.251058.119

\section{Supplemental http://genome.cshlp.org/content/suppl/2020/02/03/gr.251058.119.DC1 \\ Material}

References This article cites 49 articles, 8 of which can be accessed free at:

http://genome.cshlp.org/content/30/2/155.full.html\#ref-list-1

Creative This article is distributed exclusively by Cold Spring Harbor Laboratory Press for the Commons

License first six months after the full-issue publication date (see http://genome.cshlp.org/site/misc/terms.xhtml). After six months, it is available under a Creative Commons License (Attribution-NonCommercial 4.0 International), as described at http://creativecommons.org/licenses/by-nc/4.0/.

Email Alerting Receive free email alerts when new articles cite this article - sign up in the box at the Service top right corner of the article or click here.

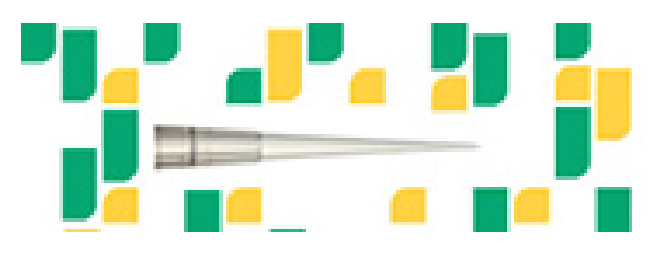

Focused on your science.

\section{Jכז}

SCIENTIFIC

suos or seisnes

To subscribe to Genome Research go to:

https://genome.cshlp.org/subscriptions 